\title{
Superconducting Wind Turbine Generators
}

\author{
Pan Yunying, Gu Danzhen \\ Shanghai University of Electric Power
}

\begin{abstract}
Wind energy is well known as a renewable energy because its clean and less polluted characteristic, which is the foundation of development modern wind electricity. To find more efficient wind turbine is the focus of scientists around the world. Compared from conventional wind turbines, superconducting wind turbine generators have advantages at zero resistance, smaller size and lighter weight. Superconducting wind turbine will inevitably become the main trends in this area. This paper intends to introduce the basic concept and principle of superconductivity, and compare form traditional wind turbine to obtain superiority, then to summary three proposed machine concept.While superconductivity have difficulty in modern technology and we also have proposed some challenges in achieving superconducting wind turbine finally.
\end{abstract}

Index Terms — superconducting, wind turbines, torque, types

\section{INTRODUCTION}

\section{A. The background of wind turbine generator}

Wind power generation technology originated in Europe, Dan Wheat, Holland, Germany and other countries where have proposed it more than 20 years. The United States, Canada and other countries also focus on wind power in recent years.

With the development of the global economy, the wind power market also develops rapidly. In the past 5 years, the world wind power market annual growth at the rate of $40 \%$. And the cost of wind power decrease increasingly. European Wind Energy Association estimated that until 2020, the cost of wind power generation will decrease to $3 \mathrm{cent} / \mathrm{KWh}$.

On the end of 2006, the world wind power installed capacity was $74 \mathrm{WG}$, while, until 2010, the value have reached $150 \mathrm{GW}$. Global wind power installed capacity in 2020 will reach 12.31 million kilowatts, which will account for the global power generation of $12 \%$. Therefore, wind power will be the mainstream for generation and become mature technology and emerging industries.

Wind power is a clean, renewable energy to generate the electricity. Although its reliability is not very well, wind turbine market has been growing rapidly. While, due to the interference of surrounding residents and low wind speed, the number of the wind power sites development are limited. On the contrary, the off shore wind power has high speeds and low interference. Therefore, it is more competitive. Nowadays, $1000 \mathrm{MW}$ wind power is under development.
The key of wind power is the wind turbine generator. Currently, the common used wind turbine generators are Double-Fed Induction Generator (DFIG) and Direct-driven Wind Turbine Generator. DFIG is most common in the market. The generators consist of the motor and the cooling system. The motor is mainly composed by the stator, rotor and bearing system. Stator winding is directly connected to the grid, the rotor windings are connected to the grid through a converter. The frequency, voltage, amplitude and phase of rotor windings' power are adjusted by the inverter according to operational requirements automatically. The unit can achieve constant-frequency generation at different speed to meeting the electricity grid and load requirements. Compared with DFIG, Direct-driven Wind Turbine Generator has no gearbox. It is a generator that direct driven by wind, also known as the wind turbine generator with no gearbox. It uses a multi-pole motor directly connected with the impeller to drive, replacing the traditional parts of the gear box. [1]

The conventional wind turbine generators have some drawbacks such as the low capacity and low economy. There are two methods to improve the wind turbine generator technology, one is improving the capacity, the other is using superconductors. Therefore the scientists put forward an idea about superconducting wind turbine generators to overcome the problems on the conventional wind turbine generators.

\section{B. Superconductor and its superconductivity}

Superconductivity is a character of certain substances that are reduced to zero at a certain temperature (generally low temperature). In 1911 Dutch physicist Heike Kamerlingh Onnes found that the mercury will suddenly be a new state when the temperature down to $4.2 \mathrm{~K}$ and its resistance is too low to measure. He called this new state of mercury as the superconducting state. And later, he found that many other metals also have superconductivity. A substance that occurs at a temperature below a certain temperature is called a superconductor.

The direct current resistivity of the superconductor is suddenly disappeared at a certain low temperature, and is called the zero resistance effect. Conductor had no resistance, current flows through the superconductor has no heat losses, therefore the current can be without resistance in a wire to form a strong current and generating a strong magnetic field. Superconducting materials and superconducting technologies have a broad application prospect. Due to the characteristics of the superconducting, more and more scientists try to applicate it in the turbine in order to improve the efficiency and advantage

open 2 access 
of wind power.[2]

\section{Merits of superconducting wind turbine generators}

The emergence of superconducting technology and its rapid development make the large-scale offshore become possible.

The characteristic of the superconducting wind turbine generators is that the power generation efficiency is high, the volume is small and the lightweight.

The low resistance and even zero resistance of HTS materials can effectively improve the power generation efficiency. In theory, the weight of the high temperature superconducting generators can be decreased to $1 / 2-1 / 3$ of the conventional motor with the same capacity, which greatly reduce wind power construction and installation cost when the high power generators placed to high meters. If the size and the weight of the generator is maintained, the capacity of the high-temperature superconducting generator can be increased several times, effectively reduce the cost of wind power generation.[2]The reason is that superconducting wind turbine generators air gap magnetic field intensity is 2 times more than the traditional permanent magnet wind turbine air gap magnetic field intensity. Improving the power density by increasing the magnetic flux density, thus the capacity of wind turbine generators can improve.[3]

Additionally, the superconducting wind turbine generators also have the advantages of low synchronous impedance, low noise, low harmonic content, simple maintenance and so on.

\section{SUPERCONDUCTING WIND TURBINE GENERATORS}

\section{A. The introduction of superconducting wind turbine}

The wind turbines rely on the input of mechanical energy. It is a machine improving gas pressure to guide the gas flow. And it is a driven fluid machine. The working principle of wind turbine is the same as the working principle of the turbine compressor. Because of the low gas flow rate and the pressure has little change, it is unnecessary to consider the changes in the specific volume of gas, so handling the gas as incompressible fluid[3].

High temperature superconducting and its application technology were developing from 1986 which has been a mature technology. Generally speaking, high temperature superconducting generators use high temperature superconducting magnets instead of ordinary copper wire coil as the excitation winding of the generators. Due to the high current carrying capacity of HTS materials (100-200 times as the carrying capacity of the same section of the copper conducting wire). Additionally, HTS wind turbine generators allow cancelling total or part of the magnetic circuit of the generators. The effective work magnetic field of the generators can reach a few $\mathrm{T}$ or more which is conducive to design a more compact, smaller generators.

The geometry of superconducting wind turbine generators depend on the comparison of operational magnetic field strength $B_{r}$ and saturation field strength of the magnet steel in the generator. The generator with $B_{r}$ lower than the saturation limit is similar to the traditional wind turbines, where the magnetic flux path is shaped by the magnetic teeth in rotor and stator. However, the effect of steel is reduced above the saturation limit and resulting the sever hysteretic losses in the steel. Therefore, when designing the superconducting wind turbine generators operating at field strength above the saturation limit of the steel, it is necessary to remove the steel in the rotor and the stator. For the magnetic field of the stator will rotate synchronously with the superconducting rotor coils, which ideally experience the time of magnetic field is constant and therefore only suffer from DC loss, so the superconducting wind turbine generators is operated as a synchronous machine[4].

\section{B. The structure of superconducting wind turbine generators}

The most common topology of a superconducting wind turbine generator is illustrated in Fig.1,which showing a multi-pole rotor based on superconducting race track coils[5].

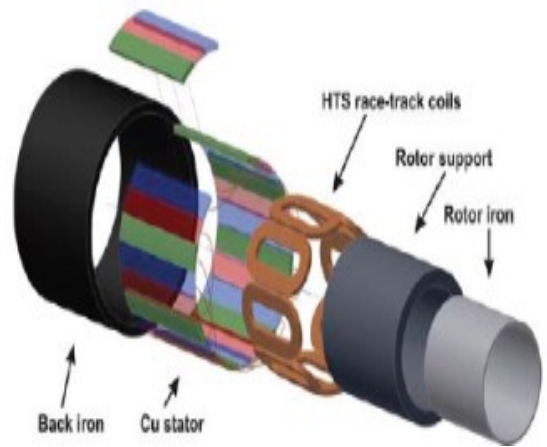

Fig.1 A superconducting multi-pole generator. Reproduced from Courtesy of Converteam.

It is a direct-drive High Temperature Superconducting Wind Turbine Generator system with low speed. The machine consists of the stator back iron, stator copper winding, HTS field coils, rotor core, rotor support structure, rotor cooling system, cryostat and external refrigerator, electromagnetic shield and damper, bearing, shaft and housing. The key of these all components is the arrangement of stator, rotor, cooling and gearbox.

The stator back iron is holding the three phase stator $\mathrm{Cu}$ winding. The coils are mounted on the rotor support structure, might holding an inner steel tube to confine the magnetic flux between the rotor and the stator and transfer the torque to the turbine shaft at room temperature.

The rotor generally contains steel laminate, concentrating the magnetic flux to the air gap and a cryostat to maintain the good thermal insulation of the superconducting coils.

\section{Design of superconducting wind turbine generators}

Conventional wind turbine has resistive windings and the iron cores are highly-multipolar machines. But, in general, the superconducting wind turbine is different. Superconducting machines requires thermal insulation between the very low temperature components and the room-temperature components. Thus, it is inevitable produce a large air gap between the superconducting field coils and air-gap armature windings. And need a pole-pitch larger than the traditional 
machines. If the pole pitch is very small, the magnetic field generated by superconducting field magnets can not reach the armature windings sufficiently.

Here introducing a size and design of superconducting wind turbine generator introduced in [2].

Fig. 2 displays a wind turbine generator designed from the Finite Element Method analysis of the magnetic field. The rotor has 12 superconducting field coils generating 12-pole magnetic field. The coils have a simple racetrack shape with a rectangular cross-section. The stator has air-gap armature windings, which are double layer, distributed three-phase windings.

Fig 3 shows the outer and inner diameters of the armature windings which is $4.32 \mathrm{~m}$ and $3.84 \mathrm{~m}$ and the outer diameter of the superconducting field coil set is about $3.6 \mathrm{~m}$. The racetrack superconducting coil is as large as $1.8 \mathrm{~m}$ (length) $\times 0.8 \mathrm{~m}$ (width) and its cross section is $0.18 \mathrm{~m} \times 0.18 \mathrm{~m}$ as shown in Fig. 4.

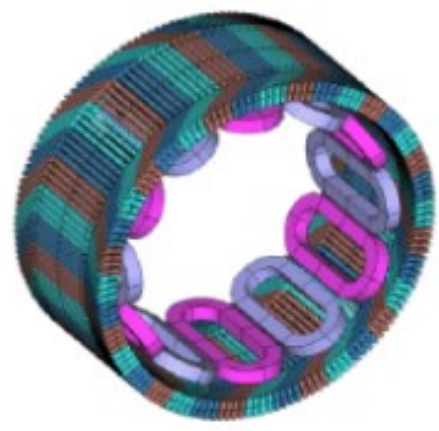

(a) Amature and field coils

Fig.2A wind turbine generator designed from the Finite Element Method Analysis of the Magnetic Field. Reproduced from reference [2]

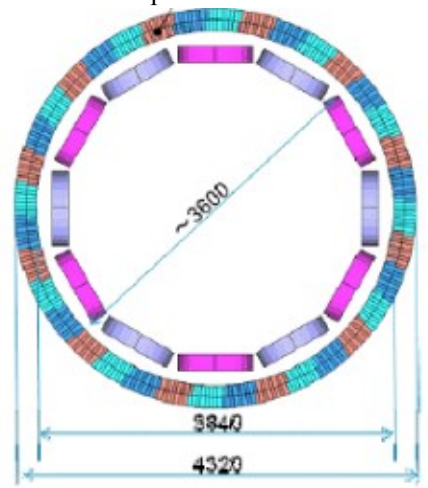

Fig.3 Dimensions of the designed superconducting wind turbine generators Reproduced from reference [2]

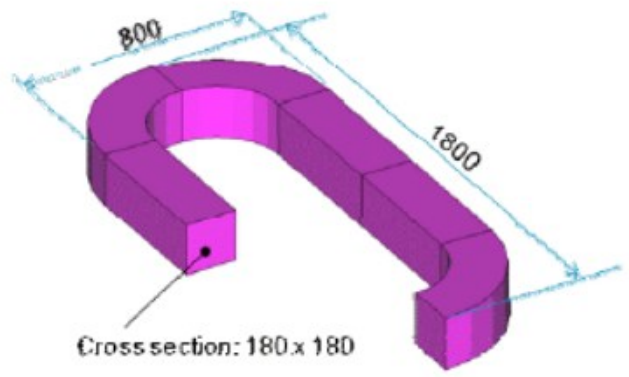

Fig.4 Superconducting racetrack coil for field magnets. Reproduced from reference[2]

\section{D. torque of synchronous superconducting generator}

The torque $\mathrm{T}$ of the superconducting generators can be determined by integrating the force $\mathrm{F}$ acting on each stator wire at air gap radius $\mathrm{R}, d T \sim R \times F$. Moreover, the Lorentz force on each wire $F \sim I_{S} \times B$.In addition, assuming the magnetic flux density in the air gap is harmonic,

$$
B(\theta)=B_{0} \cos (p \theta)
$$

$B_{0}$ is the peak flux density and $\mathrm{p}$ is the pole number. Assuming a harmonic current distribution of the stator in the coordinate system of the rotor,

$I_{S}=A_{S} \cos (p(\theta+\gamma))$

$\gamma$ is the angular displacement of the current distribution relative to the rotor field distribution and $A_{S}$ is the rms armature loading in the units of $[\mathrm{A} / \mathrm{m}]$ Therefore, we can get the torque that

$$
T \propto B_{0} A_{S} R^{2} l \cos (p \gamma)
$$

$l$ is the length of the machine.[1]

\section{DIFFERENT TYPES}

\section{A. Wind turbine with superconducting field windings}

Recently, the most common superconducting wind turbine generator is the generator with superconducting field winding

Two concepts of superconducting wind turbine generator have been proposed. First is that only field windings are superconducting. Second is that both field windings and armature windings are superconducting. Fig.5 shows the axial-radial cross-section of superconducting synchronous generators, in which the field winding is superconducting and the armature winding is copper. The superconductor is thermal insulated from ambient and cold section is equipped with torque transfer element, which can not only insulate but also transfer the torque from cold area to warm area.[1]

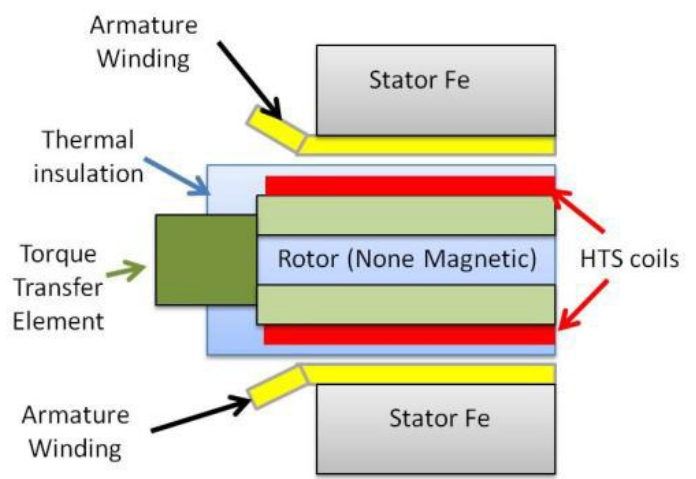

Fig.5 Axial-radial cross section of a superconducting synchronous machine. Reproduced from reference [1] 


\section{B. Fully superconducting wind turbine generators}

Fully superconducting wind turbine generators can be divided into two types. One is using LTS which used widely. The other is using HTS that only little projects use it.[1]. Compared to conventional superconducting wind turbine, the merits of fully superconducting generator is that the air gap flux density and the electric loading is increased.

Moreover, if both armature and field windings operate at the same temperature, the air gap can be much smaller. Because there is no thermal insulation between these two, the whole magnetic design of the machine can be more efficient [1]. However, the biggest problem of this concept is the AC losses that generated in the armature windings. Nowadays, because of the poor cooling efficiency, the amount of AC losses in high field $\mathrm{AC}$ application is considered as prohibitive.

\section{High Temperature Superconducting Wind Turbine Generators (HTSWTG)}

In the past, using Low Temperature Superconductors (LTS) to improve the traditional design of electric machines had been tried in many projects. And all these have shown the successful and advantages of superconducting wind turbine generators. However, there are still some challenges such as the cost of the refrigeration system and the temperature control of $2 \mathrm{~K}-4 \mathrm{~K}$ of LTS.

Nowadays, with the development and discovery of HIT materials, superconducting machines become more competitive from the commercial view. The temperature rang of HTS is $30 \mathrm{~K}-80 \mathrm{~K}$ which is much higher than LTS. Moreover, a device that using HTS has a wider temperature window, which has profound influences on the refrigeration complexity and efficiency, as shown by Fig.6.By using HTS, the refrigeration system and thermal insulation is more simple and efficiency than using LTS and MgB2.

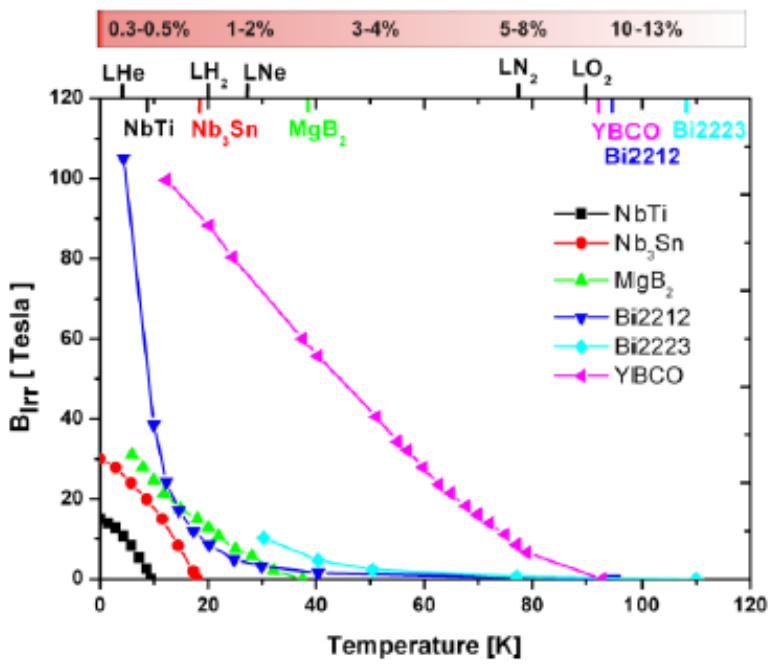

Fig.6 Operation boundaries of different superconductors, where the critical current vanishes. The top bar indicated the typical efficiency of a cryogenic cooling system. Reproduced from[6]and[7]

Actually, superconductors are rarely used in the stator because of the AC losses and it is difficult to build stator winding by HTS coils. Therefore, a radical design of a superconducting arrangement is not realistic. As a result, the HTSWTG is a synchronous generator consists of copper stator and superconductor rotor.

HTS machines can be divided to four different types[8]:

Type1. Conventional stator and HTS rotor with magnetic pole bodies.

Type2. Conventional stator and HTS rotor with non-magnetic pole bodies

Type3. Airgap stator winding and HTS rotor with magnetic pole bodies

Type4. Airgap stator winding and HTS rotor with non-magnetic pole bodies

Different types of the machines have different merits and characteristics. Due to the minimized rotor loss and does not offer substantial reduction in weight and dimension, Type1 has high efficiency. And type 2 can also get high efficiency but it needs more HTS wires to build necessary flux density. Compared with conventional stator,type 3 has higher flux density at the airgap which reduce the mass and size of machine. However, the efficiency is reduced due to the rotor iron can operate highly saturated.Type4 allows significant reduction in weight and dimension, and also minimizes potential high cost cold magnetic materials. But it requires more HTS wires in use.

Wind power is a cost sensitive market and the capital cost is important in producing large machines. For offshore wind turbines, saving cost is more important than size reduction. Therefore, the actual compromise is made between the low cost, low mass and high efficiency. For the direct-drive wind turbine generator, types 3 and 4 both can offer the lowest mass. Type 4 may be a more cost-effective module for iron and $2 \mathrm{G}$ HTS wires. Converteam use type 4 to design an $8 \mathrm{MW} 12 \mathrm{rpm}$ High Temperature Superconducting Wind Turbine Generators.

\section{PRoducED Wind TURBINE GENERATORS}

\section{A. AMSC-Seatitan}

Several machines were constructed in projects led by American Superconductor (AMSC). Currently, AMSC announced intentions to construct a direct drive wind turbine generator under the brand named SeaTitan. Based on the ship propulsion motor technology and superconducting technology of United States Navy projects, the output power of SeaTitan from AMSC can be double than the largest wind turbine generator, up to $10 \mathrm{MW}$.The key to get success is the high temperature superconducting direct drive engine with a diameter of $5 \mathrm{~m}$ and the weight is 160 t.In contrast, the diameter of a permanent magnet direct drive motor that producing the same output will reach $10 \mathrm{~m}$ and weight is also more than 200t.Moreover,the power density of superconducting wind turbine generators can achieve high power output and high economic.Fig. 7 shows 10MW Seatitan offered by AMSC. 


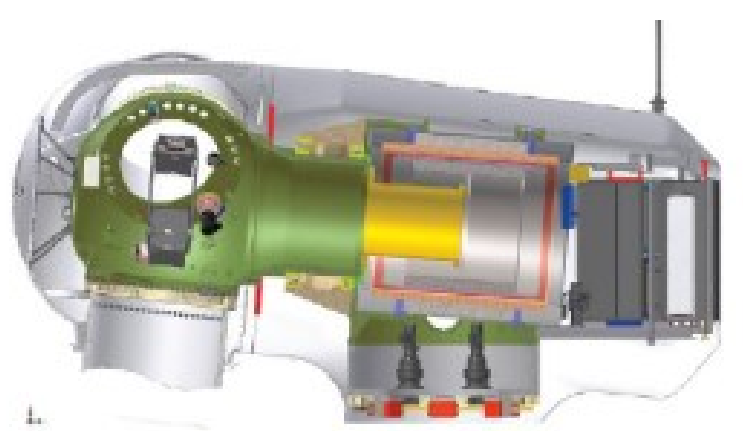

Fig.7 10MW Seatitan offered by AMSC. Reproduced from[1]

AMSC also claims that, although the focus is on the $10 \mathrm{MW}$ Seatitan system, but it is also feasible to design 20MW wind turbine generator with the high temperature superconducting technology.

\section{B. EcoSwing}

Last year, EcoSwing superconducting wind turbine generators produced by Envision and funded 1 billion yuan by EU and its member state. Significantly different from the other superconducting technologies, the power of superconducting wind turbine generator is only $3.6 \mathrm{MW}$. The purpose of EcoSwing is not the large capacity, but to promote the reduction of the cost of wind power.

Compared with the traditional wind turbine generator, the advantage of EcoSwing is that under the same torque, the weight can reduce more than $40 \%$ which can reduce the entire cabin weight $25 \%$. And the weight of other materials can reduce in proportion. At the same time, the use of rare earth will be reduced the amount of at least two orders of magnitude. Due to the advantages of EcoSwing in torque density, the economy of wind power generation will be greatly improved by the EcoSwing superconducting wind turbine generator. According to the prospect of global innovation center, the use of EcoSwing superconducting wind turbine technology are expected to decline the wind power cost by at least $30 \%$ or more. In order to make the technique applied successfully, EcoSwing project team will carry out comprehensive risk research and evaluation. After the laboratory test for EcoSwing, Envision plans to use it on the large megawatt wind turbine generators in Denmark at least one year and maintain it regularly.

"A lightweight and highly competitive superconducting wind turbine has a very exciting development potential." Anders Rebsdorf, the president of Envision in Denmark global innovation center said that EcoSwing superconducting wind turbine will be an important progress in the way of pursuit of reducing the cost of renewable energy."

Anders Rebsdorf also said that EcoSwing superconducting wind turbine technology can reduce the cost of wind power more than 30\%. And Envision hopes to use their own top technology to make the wind and solar power become the mainstream energy of the global.Fig. 8 The concept of EcoSwing.[9]

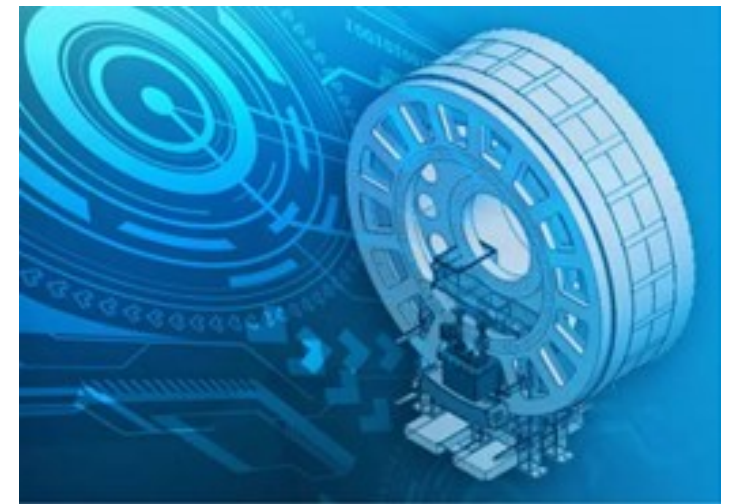

Fig.8 The concept of EcoSwing. Reproduced from [9]

\section{SUPRAPOWER}

On 20th April 2015, SUPRAPOWER held a workshop about high power electric generators for cost reduction of offshore wind. The workshop included presentations about trends on high power generators and about new developments of superconducting machines. SUPRAPOWER is funded by the European Union (EU) and it is a project that aims to a high power, lightweight and reliable superconducting generator for large offshore wind turbines. The goal is using superconductor to develop the wind power. There are nine industrial and scientific partners participate in this project. The technical physics research of university KIT will build rotating cryostat for this project. By pure heat conduction, the superconducting coil cooled to $-253.15{ }^{\circ} \mathrm{C}$. At this temperature, the superconductor does not appear the resistance phenomenon. Therefore it can realize the lossless conduction. A generator with a superconductor can be increased to $10 \mathrm{MW}$, reducing the volume and weight as well. In addition, compared to the current wide use of permanent magnet wind turbine, the superconducting wind turbine generators only need less than $1 \%$ of the rare earth. Therefore superconductors can make the wind turbine higher, more stable, simpler, saving a great deal of raw materials and reduce the construction, operation and maintenance costs, improving the service life of the turbine.[10]

Other companies also have designed different superconducting generators. However, comparing these enterprises, Sway started earliest. And the advantage of American Superconductor is that they have the core technology. Tecnalia cooperate with KIT to make the product better. Superconducting wind turbine generator is superior than permanent magnet generator. Although Sway started earliest, its development is slow and in wrong direction. American and Tecnalia \& KIT have advanced technologies. While, their technologies in wind power still need to explore. Otherwise the design and manufacture of the large-scale wind turbine generator still have some difficulties. The 3.6MW superconducting wind turbine generator produced by Envision is improved by the basis of $3 \mathrm{MW}$ wind turbine generator. Therefore, the design and production are easier. Each company has its own preponderance, the key to success is the new technology. 


\section{Challenges}

Superconducting wind turbine generator is only a concept now. The application of superconducting technology in other areas has been realized. But the application in the wind turbine generators is not quite feasible currently. Maybe there will be 5-10 years to produce a prototype or make a major technical breakthrough. For the large-scale commercial production, it is possible require $30-50$ years.

\section{A. The materials of HTS wires}

The material is the key to manufacture the superconducting wind turbine. Currently, most of use is called first generation $(1 \mathrm{G})$ wires. The ceramic superconductor is BSCCO. This kind of wires can bear the rang of the current from 100A to $180 \mathrm{~A}$ with the cross-section of $4 \mathrm{~mm} * 0.3 \mathrm{~mm}$. The manufacture process is very complex, therefore the cost is too high for application.

Recently, the second generation $(2 \mathrm{G})$ wires are also available. The ceramic superconductor is YBCO. Compared with $\mathrm{BSCCO}$, its price is lower and have more merits.

However these materials still have some problems like the supply and the price. Whether these can be in use widely is the main to consider.[13]

\section{$B$. The maintenance of superconducting wind turbine generators}

There will be high vibration when the superconducting wind turbine generators in operation. And with different wind speeds, the frequency resonant is different. Therefore, the requirement of sealing is very high. The interior of superconducting wind turbine generator is liquid nitrogen refrigeration. Once it leak, it will cause the irreparable fault on other device. On the other hand, thunder and lightning may cause the wires of wind turbine some faults. Once a failure occurs, the maintenance cost is very high because of the high technology used in the superconducting wind turbine generators. Additionally, the general staff may cannot do it for its complexity. It will affect the utilization.

\section{Excitation system}

The coils have negligible Ohmic resistance. Under normal operation, there will be a high current with a low voltage. However, when ramping the HTS coils, a large excitation voltage is required. The rang of the excitation voltage is $100 \mathrm{~V}$ or higher to achieve the field current changes within an acceptable periods of time. Therefore, a control scheme using excitation current is required. And the system must be able to operate in merely inductive load. On the other hand, HTS can not disperse the energy stored in the rotor inductance. Thus, the exciter is the key of superconducting wires protection.

Currently, two methods are in use. First is using brushes and slip rings to transfer the current. The other one is that via rotating transfer to achieve brushless excitation. This require a complex control and protection system. The first one is widely used in synchronous generators. However, the maintenance requirement is high and normally hard to tolerate. In addition, the second solution is a complex and high requirement system.
Its design is difficult. Although there have been some designed HTS, the utilization is not common.

In conclusion, the excitation system of superconducting wind turbine generators still have a lot to improve and develop. [13]

\section{Refrigeration for rotor cooling system}

Refrigeration for rotor cooling system is an important part of superconducting power equipment. The refrigeration to cryogenic temperature for HTS is different with conventional wind turbine generator. The temperature of HTS is $30 \mathrm{~K}$ in normal operation and requiring a few hundred of Watt refrigeration power. Recently, there have been no commercial applications that close to those parameters.

The best fit refrigeration is GM (Gifford-McMahon), which is regenerative refrigeration, generally developed from the cooling thermal shield of LTS MRI system, which reduce the boiloff of fluid Helium. GM used widely and many industrially established technologies provide very attractive price. However, those parameters have been designed and the operation condition is a little rough now. GM refrigerator has several drawbacks:[19]

1) GM refrigerator relies on the rotary valve to control the air flow to the cold head or return to the low pressure side of the compressor. Air flow through the valve will produce pressure head loss. Therefore, the efficiency of GM is relatively low.

2) The compressor of GM pressure is relatively stable and it can use the activated carbon to remove oil. Therefore, using commercial oil-lubricated compressor, which is reliable and having lower cost. But the compressor is large and requiring maintenance annually.

3) In addition, the cooling head includes a moving device, also need to maintain. Currently, refrigeration power of models with the temperature of $25 \mathrm{~K}$ and $100 \mathrm{~W}$ is limited. Thus, GMs have to be operated in parallel. The operation of a conventional oil-lubricated compressor depends on the orientation with respect to gravity. Thus, in some applications, it also requires other techniques.

4) Pulse Tube refrigerator is also regenerative refrigeration. In large-scale cooling capacity, the actual efficiency is low, especially cooling capacity of pulse tube refrigerators are met the problem of flow angle distribution is not uniform and interior of heat exchanger flow unsteadily, which leads to lower efficiency. And it has not been widely used in the field of superconducting machines.

The refrigerator has its own advantages and disadvantages, also affect the development of HTS. Its technology, defects and manufacturing conditions are the key factors that result the slow development of HTS.

\section{E. Stator cooling}

Effective stator cooling is the basic to achieve the effective current density of stator winding. To realize a more compact generator, a more intensive stator cooling method is required. Recently, two cooling schemes are in use. The first one is 
air-cooled stator which is more conservative. The other one is fluid-cooled stator and it is more innovative. The stator winding is completely immersed in the dielectric insulating oil. This method has been used commonly in large power generators.[13]

\section{F. Manufacturing Processes and Acceptance Tests}

Nowadays, the acceptance tests for superconducting generators have been produced as products. To reduce the production costs, developing a standardized manufacture is necessary. Compared with conventional generators, the factory acceptance tests need to be modified. Special tests are designed for special components of superconducting generators. However, this kind of tests and manufacturing process still need to be improved and develop.

\section{CONCLUSION}

. In recently years, due to more environment pollution and high carbon emission, the implementation of clean energy become popular and wind power has been the dominant position. However, conventional wind turbine generators still have some drawbacks, such as high cost and low efficiency. Therefore, many companies began to implement the superconducting wind turbine. Superconducting wind turbine generators have the advantages of high efficiency, lighter and volume is smaller. This greatly improves the preponderance of wind power. However, there are still many challenges and problems to overcome in superconducting wind turbines such as design and capacity. When these problems are overcome, it is believed that the use of superconducting wind turbine generators will become more and more common, and become a mainstream.

\section{REFERENCES}

[1] Bogi B. Jensen, Nenad Mijatovic, Asger B. Abrahamsen: "Development of Superconducting Wind Turbine Generators", Journal of Renewable and Sustainable Energy, 2013, 5(2):347-355

[2] Hiroyuki Ohsaki, Yutaka Terao, Rashidul M. Quddes, Masaki Sekino, "Electromagnetic Characteristics of $10 \mathrm{MW}$ Class Superconducting Wind Turbine Generators", International Conference on Electrical Machines and Systems, 2010:1303-1306

[3] Introduction of wind turbine. [Online]. Available: http://www.baike.baidu.com/link?url=WCmY4GUXTAUt_anNpo5mw CA1uij05gAV9ZtZ7p6OfvO6mZjyZIiz8GaezIvmYnskkKĞg7avge---o wast-KUZa

[4] A.B.Abrahamsen, N. mijatovic, E. Seiler, T. Zirngibl, C. Traeholt "Superconducting wind turbine generators" Superconductor Science and Technology, vol. 23, no. 3, 2010

[5] A.B.Abtahamsen, N.Magnusson,B.B.Jensen and M.Runde "Large superconducting wind turbine generators" Energy Procedia 2012, 24(24): 60-67

[6] H. Rogalla and P.H .Kes, "100years of superconductivity", CRC press, 2012, 24(Suppl 1):S147-S148

[7] J.Bray, "Superconductor in Application; Some Practical Aspects." IEEE Transactions on Applied Superconductivity,vol.19,no.3,pp.2533-2539,2009.

[8] Wenping Cao, High-Temperature Superconducting Wind Turbine Generators, [Online]. Available: http://www.intechopen.com

[9] Da Keke (2015,Step), What is EcoSwing, [Online]. Available: http://www.china-nengyuan.com/news/83418.html

[10] Suprapower, [Online]. Avaliable: http://www.suprapower-fp7.eu/
[11] G. Snitchler, B. Gamble, S.S. Kalsi, "The performance of a 5 MW high temperature superconductor ship propulsion motor", IEEE Transactions on Applied Superconductivity, 2005, 15(2): 2206-2209

[12] H. Ohsaki, M. Sekino, T. Suzuki, and Y. Terao, "Design Study of Wind Turbine Generators using Superconducting Coils and Bulks", International Conference on Clean Electrical Power, 2009, 234(3): 479-484

[13] J. Frauen hofer, J. Grundmann, G. Klaus, W. Nick. "Basic concepts, status, opportunities ,and challenges of electrical machines utilizing High-Temperature Superconducting (HTS) windings", 8th European Conference on Applied Superconductivity, 2008, 97(1): 182-189

[14] Zhang Hongie, Sun Jianfeng. "Application prospect of high temperature superconducting generator in wind power generation". New material industry, 2008, preface (5): 55-59

[15] ]Zheng Hailu, Jin Jianxun. "Development and research status of high temperature superconducting generators", Journal of Electronic Science and Technology University, 2007, 1673(6530):1-6

[16] Zhu Yingna (2015,April), Analysis of 10MW superconducting generator connected to grid. [Online]. Available: http://xueshu.baidu.com/s?wd=paperuri\%3A\%280946fa26130bcaa8776 $47 \mathrm{c} 268 \mathrm{ee} 6 \mathrm{~b} 6 \mathrm{c} 1 \% 29 \&$ filter $=\mathrm{sc}$ long sign\& $\mathrm{tn}=\mathrm{SE}$ xueshusource_2kduw $22 \mathrm{v} \& s c$ vurl $=\mathrm{http} \% 3 \mathrm{~A} \% 2 \mathrm{~F} \% 2 \mathrm{Fcdmd} . \mathrm{cnki} . \mathrm{com} . \mathrm{cn} \% 2 \mathrm{FArticle} \% 2 \mathrm{FCD}$ MD-10614-1015714516.htm\&ie=utf-8\&sc_us=8883208649839666566

[17] [Clive Lewis, Jens Muller. "A Direct Drive Wind Turbine HTS Generators".Wind power Monthly magazine, 2005, 13(2): pp 53-60

[18] Xu Hongling, Wang Huiling, Wang Jian, Shi Ling, Rao Rongshui, Chen Jin, Tang Yuejin, "Low temperature technology in high temperature superconducting system", Cryogenics, 2003, 2:20-24

Bi Yanfang, Hong Hui, Xin Ying, "Cryogenic cooling system and refrigerating machine for high temperature superconducting", Science China Press, 2013, 42(10):pp1001-1111

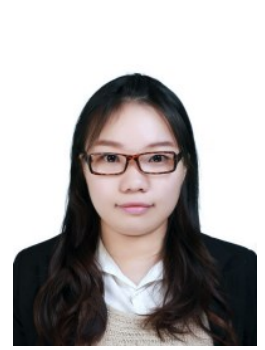

Pan Yunying was born in Shanghai, China, in 1992. She received the B.S. degrees in Power Engineering from Shanghai University of Electric Power in 2014. And now, she is a double M.S. student in Shanghai University of Electricity Power and Brandenburg University of Technology.

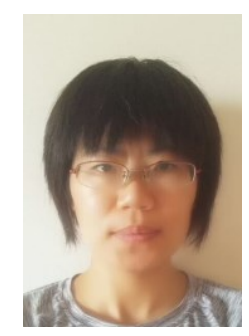

Gu Danzhen is an associate professor in Shanghai University of Electric Power. She mainly engaged in modeling and simulation of power system, power market and so on. She has participated in the study of Baosteel power grid energy management system, the transient stability study of $500 \mathrm{kV}$ Yangcheng--Huaiyin after its in operation, the research of large impact load of Jiangsu power grid and its influence and so on. She has also participated in the major program of the National Natural Science Foundation of China, such as wide area modeling and simulation theory and methods of dynamic security analysis of power system (Project No. 50595412).

She has published more than 10 papers in the Chinese Journal of electrical engineering, power grid technology, and other journals and conferences. 